Working Paper n. 35

Massimo Giannini

EDUCATION AND JOB MARKET SIGNALLING:

HOW ROBUST IS THE NEXUS?

Roma, settembre 1999 


\begin{abstract}
In this paper the Signalling approach to the explanation of wage differentials is analysed in a critical way. Departing from the classic Spence's model, the article shows how the introduction of inequalities in accessing to education leads to separating equilibria characterised by redistributive effects among workers and firms, with lower wages for high skill workers respect to the traditional model. Moreover pooling equilibria can also exist as effect of such inequalities; in this case a unique wage exists for all types of workers, operating as a redistributive effect from high-skill to low-skill workers.
\end{abstract}

J.E.L. Classification: D30 - D82 - J31 - C72

Keywords: Signalling, Human Capital, Inequality. 


\section{EDUCATION AND JOB MARKET SIGNALLING: HOW ROBUST IS THE NEXUS?}

\section{Massimo Giannini ${ }^{*}$}

\section{1 - Introduction}

The relationship between individual investment in education and wages differentials has been extensively analysed both from an empirical and a theoretical point of view; it starts from the idea that better educated people exhibit qualitative features particularly attractive for firms. The rationale for this observed regularity lies on, at least, two different theoretical approaches: the human capital and the signalling model. According to the latter, education provides a "signal" about the productivity characterising a worker randomly drawn from the population: individuals characterised by a higher level of education are in general more able than less educated people. As an example: they have lower propensities to quit or to be absent, are less likely to smoke, drink or use illicit drugs and are generally healthier (Andrew Weiss, 1995, page 133). The main idea is the following: at the birth, each individual receives a given level of "intrinsic ability" according to some exogenous (natural?) distribution; high-skill individuals are more productive, when employed, than low-skill ones and such a feature calls for a higher wage. Employers do not know, a priori, what kind of worker they are facing but education provides a key to solve the informative problem, as the pioneering Spence's work suggests, since, as in a sort of Darwinian mecha-

\footnotetext{
* Università di Roma Tor Vergata, Dip.Dir.Proc.Civ., Via Orazio Raimondo,18, 00173 Roma, E-mail: Massimo.Giannini@uniroma2.it. Acknowledgements. I am indebted to Pe-
} 
nism, only more able individuals survive to a longer staying successfully at school thanks to a lower effort. As far as the former is concerned instead, individuals acquire their productivity staying at school longer; in this case worker's productivity is solely related to length of schooling, and inborn abilities can affect marginally such characteristic. In the signalling approach hence the nexus education - individual ability is exactly reversed with respect to the human capital model; in the latter, a longer permanence at school induces a higher productivity while for the former a higher individual ability involves a higher level of education. The two sides of the coin are not necessarily rivals, and likely they are not, but it is extremely difficult to identify which of them is predominating in the real world with the result that the "human capital/signalling" debate has generated a large empirical literature. For many years the "Mincer approach" has been extensively used in empirical studies analysing the wage-education nexus hidden in the human capital model; nevertheless recent contributions, especially from Altonji, (1995), have shed further light on the topic taking into account for agents' heterogeneity measured by several individual factors, as parental income, parental education, individual ability, place of birth and quality of the attended school, as in Card and Krueger, (1996), other than variables related both to individual and socio-economic context. Main result of these empirical studies underlines the signalling - or at least they arise some serious doubts on the human capital explanation to the observed regularities - rather than the human capital explanation to wage differentials; under this point of view the empirical analysis does not provide a definitive key to solve the

ter Sorensen and Anthony Atkinson for helpful comments on a previous version. Usual dis- 
theoretical debate. For such reason in this paper we shall try to analyse the signalling approach robustness in providing an explanation to the wage differentials from a pure theoretical point of view. Obviously it is out of my goals any attempt to provide the debate with a conclusion; rather I will to focus on some logical and theoretical embarrassment of the signalling model, although I am aware that the theoretical model is only a stylised representation of a complex reality. Our analysis starts from the idea that in the signalling approach, hereafter identified with the Spence's model and following refinements ${ }^{1}$, it is implicitly assumed that individuals have a free access to education and only a subjective reason (the personal aversion towards education) "constrains" their choice. But if education has a cost, and if individuals face a budget constraint, is it still possible to achieve the education level signalling properly own productivity? In the Spence's model each individual owns the same opportunity of accessing to education; if we abandon such an assumption and we take into consideration inequalities among individuals to access to education then several questions arise about the validity of the signalling approach in explaining the earnings distribution, as the paper points out. The article is composed as follows: in section 2 a brief review of the Spence's model is provided. It underlines the "separating property" of the solution, obtained by suitable refinements of the Nash equilibrium, due to Cho-Kreps, (1987), and Banks-Sobel, (1987). In section 3 we

\footnotetext{
claimers apply.

${ }^{1}$ It is worth stressing that in the following we are going to focus on the "signalling" rather on "screening" approach; the difference is in the first move: in the signalling approach the worker plays the first move while in the screening one such move is left to the firm by means of wage offers. An interesting case of screening in a dynamic framework is provided by Noldeke and Van Damme, (1990).
} 
shall use the same framework but with a "rationed agent" who can not properly signal her quality to the firm. We shall see that both separating and pooling equilibria are possible, with a particular result about the way more able individuals are remunerated in a separating equilibrium. Conclusions follow.

\section{2 -The Spence's model: a review.}

The signalling approach takes into consideration the "strategic interaction" between individual and firm on the job market; such interaction can be well analysed by the theory of Signalling Games because of the informative problem induced by the theoretical framework. In fact when a firm faces a worker on the job market, it knows, a priori, neither the productivity of the latter nor it can hope that the individual "reveals" herself, since less-skilled individuals strictly prefer "to mimic" skilful types in order to get higher wages. Hence an "asymmetric information game" is at the work here; there is a player (the worker) who owns a larger information set than her opponent (the firm), since the former knows her type but not so for the latter. In order to keep the discussion as intuitive as possible, I am going to analyse the simplest case of signalling game. There exist two "types" of worker with different ability indexed by the integer $t$, viz. low-skill $(t=1)$ and high-skill $(t=2)$. The opponent is a firm 2 asking for the services of the workers. Workers know their type but not so the firm; the latter observes only the education level chosen by the worker. Both players are maximizer: workers want

\footnotetext{
${ }^{2}$ We assume for simplicity a single representative firm, but the result does not change if we assume two firms competing à la Bertrand.
} 
to maximise their utility function choosing an education level $e \in(0, \infty)$, given their type, $t$, and the expected wage $w$ paid by the firm. The latter is a profit-maximizer, hence pays a wage equal to the worker productivity (ability). But the firm does not know - a priori - such a characteristic; it can only set up a beliefs system about it on the basis of the observed education level. Workers know this and in turn choose the education level performing conjectures on the firm response (wage) to the chosen education level. So both players, firm and workers, base their choices on some beliefs about the opponent's strategy. Before describing in detail the property of the beliefs system, we return to the workers' preferences. For each type $t=1,2$, arguments of the utility function are the wage, $w$, and the education, $e$, so both types can select a bundle $(w, e)$ in the $\mathfrak{R}^{+} \otimes \mathfrak{R}^{+}$space. Individuals are characterised by complete, reflexive and transitive preferences on the bundles $(w, e)$ and so ordering is possible. This property allows us to work with a continuous, concave utility function which is strictly increasing in $w$ and strictly decreasing in $e$. Moreover, since type 1 is more "education-averse" the curvature of her utility function is in any point greater than type 2 . This set of assumptions can be summarised by two families of indifference curves in the $(w, e)$ space, one for each type. Such curves are continuous and increasing, but the curves of type 1 are in any point steeper than type 2, so, when they cross, they cross once ${ }^{3}$. Finally, utility is increasing from South-East to

\footnotetext{
${ }^{3}$ This single-crossing property is obviously crucial for the result, although quite reasonable, and it has several implications, not last the fact that the supports of the signals (education in our case) sent by various types are increasing in the type. This does not preclude pooling, but a pool must be an interval of types pooling on a single signal, with any type in the interior of the interval sending only the pooling signal. (Kreps-Sobel, 1994, page 855).
} 
North-West in the $(w, e)$ space. We shall find very useful analyse the equilibrium by these indifference curves.

Now we return to describe the game. With $t=1,2$, we identify types; $p(t)<1$ means the fraction of type $t$ in the workers population and it represents the (prior) probability to engage a type $t$ in absence of additional information; this is common knowledge. Workers have a te productive value for the firms, $t \in\{1,2\}$ so that, in a full information equilibrium, firm pays $w(e)=e$ to less-skilled individuals and $w(e)=2 e$ to more able ones. The problem consists in recovering such a result in the asymmetric information context, i.e. when the firm does not know, a priori, what type is facing. However, it is clear that the firm is never willing to pay a wage above $2 e$ and, likewise, workers do not accept a wage below $e$. In other words, it is common knowledge that $e \leq w(e) \leq 2 e$. Each type is characterised by a conditional probability distribution $\rho(e \mid t)$; the latter represents the probability that a type $t$ chooses $e$ as the educational level. The firm responds to the education level $e$ following some beliefs system $\mu(t \mid e)$ representing the probability, assigned by the firm, that the observed education level $e$ comes from a $t$ type. On this belief, the firm chooses its best-reply, viz the wage $w$. It remains to define how the firm sets up beliefs and the proposed solution in literature is the following: for each education level $e$ chosen by the worker with positive probability, $\mu(t \mid e)$ follows the Bayes rule:

$$
\mu(t \mid e)=\frac{p(t) \rho(e \mid t)}{p(1) \rho(e \mid 1)+p(2) \rho(e \mid 2)} \in[0,1]
$$


Equation (1) shows the conditional probability $\mu(t \mid e)$ as a ratio between a joint probability (numerator) and a marginal probability (denominator) ${ }^{4}$. In this way the expected wage follows:

$$
w(e)=\mu(1 \mid e) e+\mu(2 \mid e) 2 e
$$

As it is easy to check, $e \leq w(e) \leq 2 e$. For example, if the firm believes that the observed education level $e$ comes from a type 1 , then $\mu(2 \mid e)=0$, $\mu(1 \mid e)=1$ and the firm best reply is $w=e$. Nevertheless Eq. (1) can be used only if $\rho(t \mid e) \neq 0, t=1,2$, i.e. when the denominator is different from zero, which occurs for education levels chosen in equilibrium with a positive probability; nevertheless, in order to test an equilibrium robustness, we need to analyse "off-equilibrium" education levels for which (1) does not hold, since the denominator is, in this case, zero - off-equilibrium moves are, by definition, not chosen by players hence their probability to be played is zero. To avoid such a problem, we have to set up a beliefs system also for offequilibrium moves. The equilibrium refinement proposed by Cho and Kreps, (1987), known as Intuitive Criterion, fits this end ${ }^{[}$.

Now we are ready to show the game solution through the diagram in Figure 1 This figure shows a "separating equilibrium" where type 1 chooses the education level $e_{1}$ and type 2 chooses $e_{2}$. Such a result can be supported by beliefs putting zero weight on the fact that education levels $e<e_{2}$ come,

\footnotetext{
${ }^{4}$ It might be worth spending some words on it. The numerator is a joint probability because it is the product of a marginal and a conditional probability; it represents the probability that an individual $t$ chooses the education level $e$ since $p(t)$ is the (marginal) probability to face a $t$-type and $\rho(e \mid t)$ is the probability that type $t$ chooses the education level $e$. The denominator represents the (marginal) probability to choose an education level $e$ whatever the type.

${ }^{5}$ For a two types game, this refinement corresponds to the Banks-Sobel' Divinity.
} 
in equilibrium, by type 1 ; in this way $\mu(2 \mid e)=0, \mu(1 \mid e)=1$ and the firm "best reply" is $w=e \forall e<e_{2}$ according to (2). Given such a firm reply, type 1 selects $e_{1}$ in order to achieve her first best; note in fact that in $e_{1}$ the indifference curve shows a tangency with the ray $w=e$. In such equilibrium, educational levels above $e_{2}$ reveal exactly type 2 because $\forall e>e_{2}$ type 1 is worse off than in equilibrium, i.e. in $e_{1}$. As the diagram shows indeed, such education levels lie under the indifference curve of type 1 characterising the equilibrium. According to such reasoning, if the firm observes an education level lying in this region, i.e. $\forall e>e_{2}$, it can set $\mu(2 \mid e)=1, \mu(1 \mid e)=0$ and

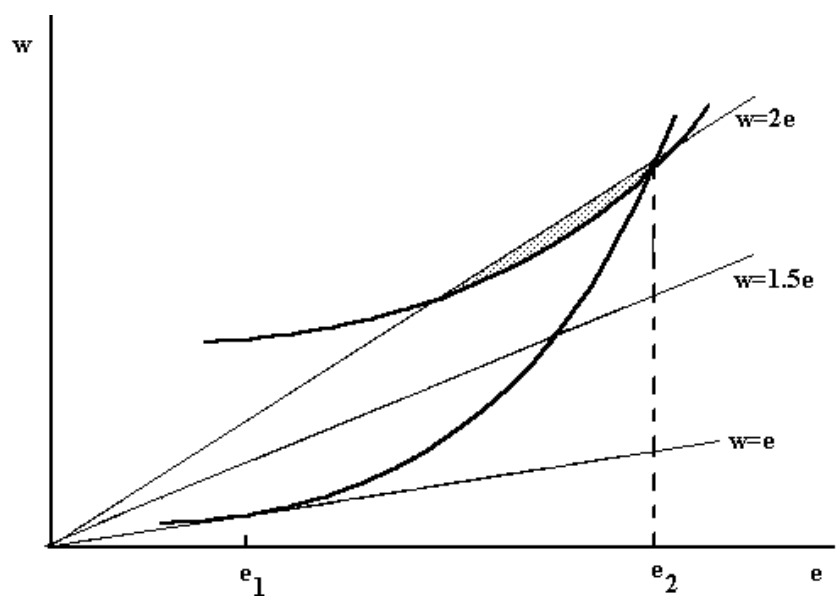

Figure 1 - A Separating Equilibrium

$w=2 e$, according to $(2)^{6}$. But then the best choice type 2 can do is to signal $e=e_{2}$ which provides her the maximum feasible utility (as we remarked, for

${ }^{6}$ It is worth noting that the belief $\mu(2 \mid e)=1$ for $e=e_{2}$ holds although type- 1 is indifferent w.r.t. $e=e_{1}$. Such a belief is supported by the Divinity Criterion; roughly speaking such re- 
$e<e_{2}$, type- 2 can not be properly identified). Nonetheless such a choice does not assure the first best; as the figure shows, the type 2 indifference curve intersects the ray $w=2 e$ for $e=e_{2}$. If we were in a full information context, type 2 would achieve a first best solution with a lower education level than $e_{2}$. The presence of asymmetric information produces an inefficiency characterised by over-signalling; in other words, type 1 can freely perform her maximising program while type 2 must take into consideration the separating constraint. Now we have to demonstrate that only separating equilibria are possible in such a game. Let us consider a point $e$ in the shaded region in Figure $1^{\text {Q }}$, despite this region is above both indifference curves, hence representing strictly preferred bundles w.r.t. the equilibrium situation, it is not take into consideration by the workers. In fact if the firm had observed an education level lying in this region, then it would not have could exclude any type since both workers would gain by sending this signal. If we assume that types are randomly drawn from a uniform distribution, i.e. $p(1)=p(2)=1 / 2$, the more reasonable beliefs system is $\mu(1 \mid e)=\mu(2 \mid e)=1 / 2^{8}$, then the firm best reply would be to offer $w=1.5 e$

finement establishes that observing a given signal in equilibrium - $e_{2}$ - for which type-1 gets the same utility level of a lower signal $-e_{1}$ in our case - while type- 2 has a strong incentive to depart from such a message in order to be properly separated, then we can attribute the observed signal to type-2 with probability one. See Banks and Sobel, 1987.

${ }^{7}$ It is worth stressing that these points are off-equilibrium, hence we can not set up a beliefs-system by the (1), viz we can not assume $p(1) \rho(e \mid 1)+p(2) \rho(e \mid 2)>0$. We are going to use the above mentioned Nash refinements in order to restrict beliefs.

${ }^{8}$ Such a belief is coherent with the Bayes rule because if the observed level $e$ were the equilibrium result, then we necessarily should have $\rho(e \mid 1)=\rho(e \mid 2)=1$ which, together with the prior probability $p(1)=p(2)=1 / 2$, provides exactly the above belief. Moreover it is not by chance that the posterior probabilities (belief) match the prior probabilities, since, in absence of a new information on types, the Bayes rule provides no updating on beliefs. 
(by (2)). Workers know this firm strategy and they choose a common education point $e \in\left(e_{1}, e_{2}\right)$ on the mix-line $w=1.5 e$, since this is the better choice in such a situation. In other words seems that our original separating equilibrium collapses in a pooling equilibrium but this would be a wrong conclusion. In fact in a pooling equilibrium type 2 is worse off than in the separating one, since the pooling involves lower indifference curves for type 2 . Hence she has a strong incentive to deviate from the pooling equilibrium by sending a higher signal in order to separate properly herself with the result of breaking down the pooling equilibrium; in such situation the best strategy for type 1 is $e_{1}$ since it can not mimic a type 2 .

Concluding, in the Spence's model only a separating equilibrium survives, solving the informative problem thanks to a workers self-selection by means of education. Despite education does not increase individual ability at all, it provides the right signal to identify the worker productivity. The model offers a simple but powerful microeconomic theory justifying a positive relationship between wage and education, which in turn hides a positive relationship between wage and individual productivity.

\section{3 - A slightly different game.}

So far the story sounds quite standard. However, as pointed out in the introduction, the Spence's model does not involve any pecuniary cost for education; such a characteristic leads implicitly to assume that either education is entirely free or individuals are rich enough to achieve the necessary educational level. In this section we are going to modify the Spence's model by assuming that education is costly (not only as individual effort) and in 
order to analyse the effect of such an assumption let us introduce a third worker. The latter is a high skill worker but she is poorer than the typical type 2 ; we shall call, conventionally, this "rationed" agent as $2^{1}$ and the nonrationed one as $2^{2}$. The only difference between these types is in the feasible education set, viz, while $2^{2}$ selects $e \in[0, \infty)$, type $2^{1}$ can choose only in the $e \in[0, \bar{e}]$ domain - with $\bar{e}<e_{2}$ - because of her income constraint. It must be remarked that there is no borrowing in the model and this for two orders of questions: firstly because we are investigating the robustness of the signalling approach to introduction of inequalities among workers and in particular to heterogeneity in accessing to education. Secondly because, as it will be clearer later, borrowing does allow rationed individuals to achieve the necessary education level, but, because of the debt refunding, they incur in a loss of utility with respect to non-rationed players. In other words, in the separating equilibrium, there is a redistributive effect from the rationed worker to the lender; the introduction of borrowing does not preserve from this form of market failure. Under this point of view, the assumption of borrowing does not change our conclusions about the failure of the market in providing a wage distribution which is solely related to inborn abilities ruled by Nature, as we are going to point out. Finally several authors have stressed the incompleteness of the human capital market because of the lack of collateral. By so doing we restrict the feasible message set for player $2^{1}$ to a compact one with the right bound lower than the signalling threshold $e_{2}$. We have now to investigate how such an assumption can affect the previous 
section results; we distinguish two cases: in the first one $\bar{e}<e_{2}$ but $\bar{e}>e^{*}$ and $\bar{e}<e^{*}$ in the second one (see Figure 2 below where only type 1 curve

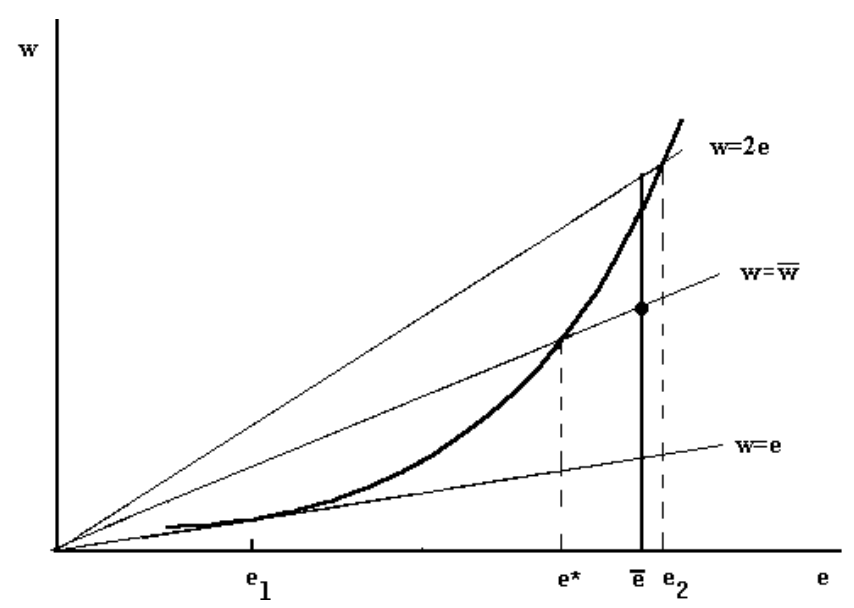

Figure 2

has been drawn). In the Spence's model, because of assumptions on the individual preferences, high Sender (workers) are stronger than low ones; The higher $t$ is, the more $R$ (the firm) is willing to pay. Consequently, ... any Sender type $t$ would like $R$ to believe that $t=T$ (the highest type) ... higher types are more willing to send higher signals than lower types' (Cho-Sobel, (1990), page 392). In other words, in such a signalling game, the highest type - type 2 in our case - is always better off in a separating rather than in a pooling equilibrium, since she can achieve a higher indifference curve. For such a reason this type of sender prefers revealing properly herself by means of education and lower types try to mimic, until possible, her behaviour; this is exactly the way by which we broke down the pooling equilibrium in the 
previous section: the highest type is always willing to choose any necessary amount of education separating herself from the lowest one. For such a reason, type $2^{2}$ does choose $e_{2}$ as education level and the informative problem affects only type $2^{1}$; in other words, we have only to concentrate on players 1 and $2^{1}$ who are the only agents sending a signal $e<e_{2}$.

When the highest type is constrained to a compact message set, pooling equilibria are also possible; this is one of the contributions of the Cho and Sobel article, Proposition 4.1 page 395. For a rigorous demonstration of such a result the reader is urged to read the cited article; nevertheless an intuitive justification can be provided on the basis of the previous reasoning. Since the highest type has always an advantage in separating rather than in pooling equilibria, she does choose the maximum education level signalling properly herself but the latter could require a value outside the feasible choice set. In this case the feasible message set is not sufficient to separate workers, but the optimising program induces the highest type to use the maximum feasible education level in the attempt to signal her productivity; by so doing she will be mimicked by the low-skill individual who tries to get a higher earning. The final result is a pooling equilibrium surviving the mentioned Nash refinements.

By the above reasoning we obtain an interesting result: when individuals, and in particular high-skill types, can not access to a suitable education level and a pooling equilibrium arises, then education does not provide any explanation to the earnings distribution, at least in this class of models. Moreover, in a pooling equilibrium, part of more able individuals "ability rents" (I borrow this terminology from Stiglitz, 1975) is redistributed to less 
able ones, since the latter get a higher wage than in a separating equilibrium. In other words we assist to a market failure operating as a tax on high-skill individuals and accruing to low-skill ones as a subsidy.

It must be remarked however that separating equilibria are still possible; let us come back to our example beginning from the $\bar{e}>e^{*}$ case. In such a case a pooling equilibrium could be represented by the bold dot in Figure 2 on the $\bar{w}$ ray $^{9}$ - the maximum feasible signal, as suggested by the cited work of Cho and Sobel; yet such an equilibrium can be easily broken down. If we mark $u^{*}(t)$ the utility achieved by type $t$ in this pooling equilibrium, it is easy to note that $u^{*}(1)<u\left(1, e_{1}, e_{1}\right)$, i.e. type 1 has an incentive to deviate from the pooling equilibrium choosing $e_{1}$, since such a value corresponds to a higher indifference curve than $u^{*}(1)$. She prefers reveal herself as a low-skill worker and receive the "right" firm response to such a signal, i.e. $w=e_{1}$. In other words, we obtain a separating equilibrium once more, where type 1 chooses $e_{1}$, type $2^{1} \bar{e}$ and type $2^{2} e_{2}$. Such a result is still possible to the extent that $\bar{e}>e^{*}$; when $\bar{e}=e^{*}$, i.e. when type 1 is indifferent between $e_{1}$ and $\bar{e}$, a different behaviour arises. Let us consider Figure 3. In such a situation, type 1 is indifferent between separating herself at $e_{1}$ or pooling at $\bar{e}$; the behaviour of type $2^{1}$ depends instead on her indifference curve. If the latter cuts from below the ray $\bar{w}$, for $\bar{e}=e^{*}$, as in Figure 3,

\footnotetext{
${ }^{9}$ This is the wage corresponding to $p(1) e+p\left(2^{1}\right) 2 e$ where $p()$ is the frequency of types 1 and $2^{1}$ in the workers population.
} 
then type $2^{1}$ can achieve a higher indifference curve in a pooling equilibrium for some $e<e^{*}$. Obviously type 1 will follow such a choice, since in this case she achieves a higher indifference curve than in a separating equilibrium.

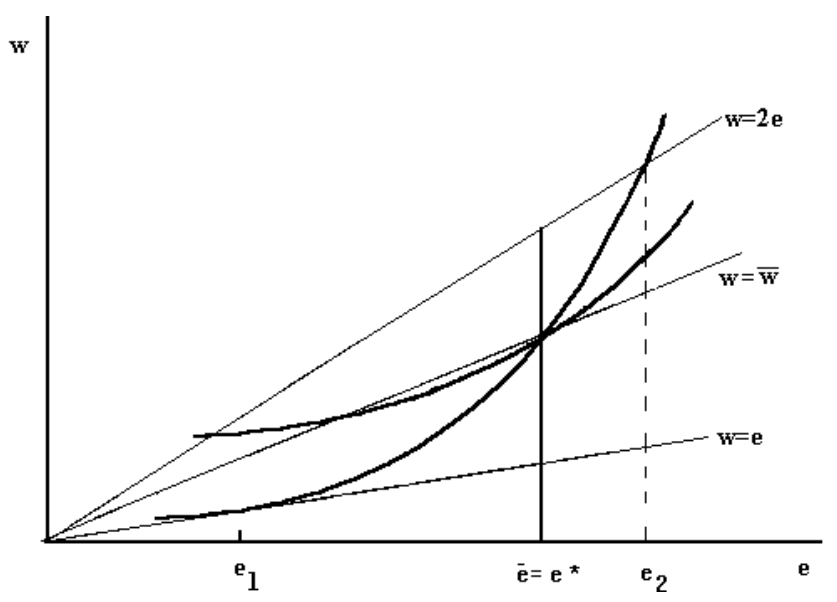

Figure 3

If instead the indifference curve of type $2^{1}$ cuts from above, or is tangent to, the $\bar{w}$ ray for $\bar{e}=e^{*}$ then type $2^{1}$ is worse off in any other point than $\bar{e}$ while type 1 is indifferent between $e_{1}$ and $\bar{e}$. Hence signalling $\bar{e}$ is more important for type $2^{1}$ than type 1 and for such reason we can assign weight one to the belief that $\bar{e}$ is coming from the former rather than the latter - the argument follows the Divinity criterion, as previously remarked. In this case hence we obtain a separating equilibrium once again but it is also the left bound of the separating equilibria set; at the left of $e^{*}$ in fact, only pooling equilibria are possible at the maximum feasible signal $\bar{e}<e^{*}$ (this is a case 
where the Cho-Sobel proposition 4.1 applies) since this kind of equilibrium involves higher indifference curves for both types, and in particular for type 1 which has a strong incentive to deviate from the separating equilibrium. In other words, when $\bar{e}<e^{*}$ the maximum feasible signal sent by type $2^{1}$ is no longer sufficient to prevent mimicking from type 1 and a pooling equilibrium arises.

The result obtained shows that, depending on the $\bar{e}$ value, it is possible to identify either a separating or a pooling equilibrium; these equilibria sets are parameterised to $\bar{e}$ and, varying the latter, we obtain a continuum of equilibria spanning from pooling to separating; in other words, the Nash equilibria set for such a game is connected.

So far, we have intentionally skipped any reference to the firm best reply to type $2^{1}$ in a separating equilibrium, i.e. when $\bar{e} \in\left(e^{*}, e^{2}\right)$; now we fill the gap. In a separating equilibrium - as the one where type 1 chooses $e_{1}$ and type $2^{1}$ chooses the maximum feasible signal $e^{*}<\bar{e}<e_{2}-$ type $2^{1}$ is properly identified as a high-skill worker but the firm best reply can not be $w=2 \bar{e}$, because in this case type 1 has an incentive to deviate from the equilibrium, given that the bundle $(2 \bar{e}, \bar{e})$ lies on a higher indifference curve for this kind of worker (see figure 4 below).

In such a situation the firm best reply to type $2^{1}$ is to fix a wage level compatible with the separating equilibrium, as the wage $w^{*}$ corresponding to the bold dot in Figure 4. Note that such a wage level is lower than the one type 
$2^{1}$ should receive: $w^{*}<2 \bar{e}$; nonetheless this is the best that both players can do. The constraint on the type $2^{1}$ signals set, jointly to the presence of a type 1 , produces an inefficiency in the job market in sense that type $2^{1}$ is paid less than her productivity; firms hiring this kind of worker attain positive profits in equilibrium thanks to the lower ability rents accruing to type $2^{1}$ workers 10 .

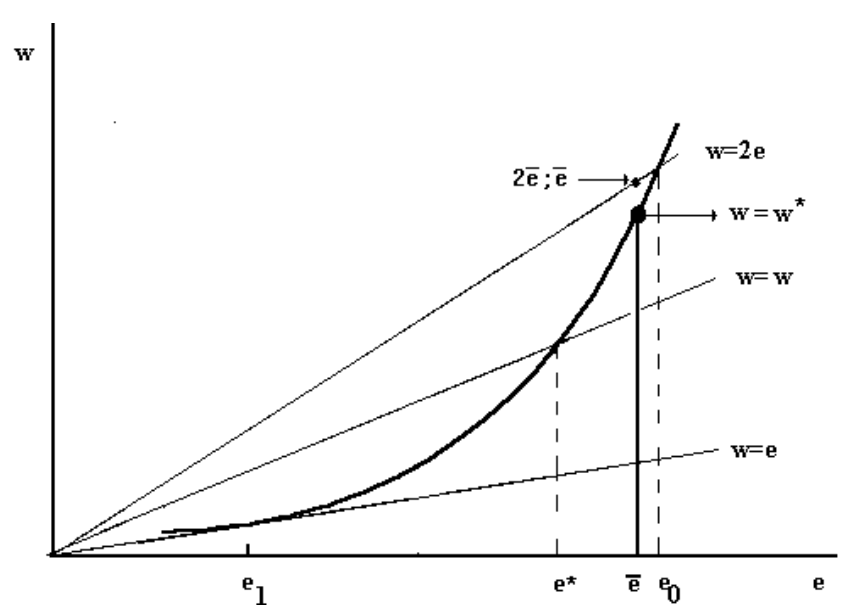

Figure 4 -A Different Separating Equilibrium

Moreover if there exists, as previously assumed, a second high-skill worker who can freely choose the properly separating education level, then another theoretical embarrassment arises, viz. the presence of two equally productive individuals receiving a different wage. If we assume that the economy consists of two sectors, one using low-skill and the other one highskill workers, then in the "high-tech" sector firms strictly prefers type $2^{1}$ to

\footnotetext{
${ }^{10}$ This is a very particular form of "equilibrium" since firms will compete to each other for hiring this particular worker. Nevertheless this competition can not increase the wage beyond $w^{*}$ for the reasons we have said.
} 
$2^{2}$ since the former owns the same skill level but she is willing to accept a lower wage than her productivity level, since she can not do better. It is clear that in such a situation no firm demands for type $2^{2}$. The only way for the latter not to be expelled from the labour market is to mimic type $2^{1}$ behaviour, but then the only separating equilibrium surviving is the one characterised by the bundles $\left(e_{1}, e_{1}\right)$ for type 1 and $(2 \bar{e}-\delta, \bar{e})$ for both types 2 , where $\delta$ is a part of the more able workers ability rents accruing to the firms because of the presence of rationed individuals high-skill individuals to firms is as high as $\bar{e}$ converges from the right to $e^{*}$, i.e. as inequalities in accessing to education are high.

The results obtained so far underline that a separating equilibrium operates in a proper way when education is a type of commodity that individuals can purchase in any desired amount; when inequalities in accessing to education are introduced in the Signalling approach, then this theoretical scheme arises a series of market failures characterised by redistributive effects among players. Pooling equilibria involve a partial transfer of ability rents from high-skill to low-skill workers. Even worse, in such a case the signalling approach fails to provide an explanation to wage differentials. Viceversa, in a separating equilibrium recipients of the redistributive effect are the firms. In both cases, some workers receive an earning which is less than their productivity.

\footnotetext{
${ }^{11}$ This kind of inefficency does not arise in a pooling equilibrium; in this case in fact type $2^{1}$ is grouped with type 1 and type $2^{2}$ can be properly identified, receiving the right wage.
} 


\section{Conclusions}

Goal of the paper was to analyse the robustness of the signalling approach in explaining the nexus wage - education. In section 2 we analysed the Spence's model underlining the separating property of the unique equilibrium surviving the suitable Nash refinement. In section 3 we altered a key assumption of the model by introducing heterogeneity among more able individuals, showing how inequalities in accessing to education lead to a serious distributive problem.

It seems to me that three main conclusions arise. The first one concerns inequalities in accessing to education; if a part of more able individuals can not send the right amount of signal, then they suffer of a utility loss which induces a redistributive effect to the firms. The consequence is that each more able individual, rationed and not, is underpaid with respect to her productivity. Moreover it is not possible to rule out such a distortion by subsidising individuals, since, because of the asymmetric information, we do not know, a priori, the recipients. Neither borrowing solves the problem, since it allows individuals to attain the necessary education for a right signalling, but in equilibrium such individual gains the same wage of the nonrationed individual with an additional cost due to refund; also in this case there is a partial redistributive effect of the borrower ability rents to the lender, despite the presence of a fully separating equilibrium. The only way to avoid such a problem is to guarantee to everyone a free access to education.

The second point concerns the real functioning of an educational informative channel. There exist several external factors which can reduce 
strongly this kind of communication. When such factors make noisy the communication of the individual ability through schooling, the separating device does not work properly and more able individuals can be grouped with the less able ones and viceversa. In this case there is not only a private effect but also a social one, since, as noted by Stiglitz, (1975), a productive line composed by individuals with different abilities is less productive than two distinct lines characterised by homogenous workers. The presence of external factors induces a wide range of miss-allocation effects, altering the sorting mechanism.

The third and last point takes into consideration the trade-off between efficiency and equity. In the microeconomic model used so far, a $t$-type individual who studies $e$ has a value te for the firm. Although education does not affect the individual ability at all, a sort of learning by doing is at work here, since individuals' productivity depends both on $t$ and $e$. More precisely, the signalling device involves a "by-product" to the extent that more able individuals must invest considerable resources in education in order to be properly separated; the final result is that they are more productive not only because they are more able but also because they stay longer in school. From this point of view, the learning component produces a level effect in the aggregate production as consequence of the signalling device. If argument of a social utility function were the level of GDP, then any policy increasing the amount of education necessary to provide a fully separating mechanism should be considered. In fact, from a macroeconomic point of view, a perfect signalling - hereafter identified with the model of section 2 increases the aggregate output, since it establishes a monotonically increas- 
ing relationship between individual ability and amount of education, $e(t)$, $e^{\prime}(t)>0$, where $t$ is the individual ability. In general, we can define the aggregate output, when the separating device works, as: $Q_{s}=\sum_{t=1}^{T} t e(t) \pi(t)$, where $t=1,2 \ldots T$ is the type and $\pi(t)$ the frequency of type $t$ in the workers population. It is clear that if the separating device does not work at all $e(t)=e_{p} \in\left(e_{1}, e_{T}\right)$, then $Q=e_{p} \sum t \pi(t)<Q_{s}$, where $e_{p} \in\left(e_{1}, e_{T}\right)$ is a common education level chosen by types as "best reply" to the mean wage rate $\sum t \pi(t)$ offered by the firm. Moreover, in such a situation, the earnings distribution collapses to a Dirac-delta function, i.e. to a zero dispersion function around $\bar{e}$. On the other side, the separating device induces the highest dispersion among earnings and the direct consequence of such an unpleasant result is that more able individuals have a greater weight in the aggregate output. In other words, higher investments in education induce stronger inequality and vice versa; if engine of the growth is human capital, then the higher inequality, the faster accumulation.

The distributive question related to the earnings distribution is particularly relevant in the real economy. As underlined by Rebecca M. Blank (NBER Reporter, Fall 1996, page 11), during the 80's, wages for less-skilled workers declined steadily. Between 1983 and 1989, GDP growth of 1 percent was correlated with a $\$ 0.32$ decline in weekly wages for the poorest decile of the population....Among men with less than a high school diploma who work full time all year, real weekly wages declined 22 percent between 1979 and 1993...In short, for the last 15 years, economic growth has not 
been as powerful in fighting poverty as it was in the past. A possible explanation for such a result can be provided by the signalling approach, where more able individuals play a leading role in the production process and consequently in the distributive one; although less-skilled workers choose voluntarily a low education level according to their preferences, if signalling works fully, they tend to have a low weight on the growth process, being the latter primarily driven by more able workers, with the result that, in a growing economy, the weight of less able individuals tends to be reduced. One can object that the distributive problem involved in the signalling model is a false matter: in the Spence's model the individual's inborn ability is solely related to a genetic, or natural, endowment so that the earnings distribution obtained in a separating equilibrium reflects only genetic differences among individuals in a fair way. For such a reason several authors underlines the fairness of such result. Nevertheless, a completely different interpretation exists (see Atkinson, 1980, among others): inborn ability is not totally exogenous since it is related to parental skill, family background, standard of life which are intimately connected to the socio-economic context. These factors represent a source of mistake in sorting individual so that the more the signalling is "noisy", the more the distributive process is biased; in such a case not only the market fails to allocate workers according to their real ability, but it creates additional inequalities. The debate about the equity characterising a "natural" distribution is an old matter and a too complex subject to be discussed here. This work simply wants to underline some theoretical inconsistencies involved in the signalling approach to wage differentials. To the extent that inequalities in accessing to education and in in- 
born abilities induced by different parental contexts reduce greatly the informative power of education, the signalling approach arises more questions than answers. Obviously this does not mean that a signalling device does not operate at all, but simply that the power of such an approach in understanding the nexus wage - education has to be carefully considered.

\section{REFERENCES}

Altonji, J. G. 1995. "The Effects of High School Curriculum on Education and Labor Market Outcomes," Journal of Human Resources, 30:3, pp. 409438.

Atkinson, A.B. 1983. "Income Distribution and Inequality of Opportunity," in Social Justice and Public Policy. Atkinson, A.B., Eds. London: Wheatsheaf Books, pp. 77-93..

Banks, J.S. and J. Sobel. 1987. "Equilibrium selection in Signaling Games," Econometrica, 55:3, pp. 647-662.

Blank, M. R. 1996. "Labor Markets and Public Assistance Programs", NBER Reporter, Research Summary, Fall, pp. 11-13.

Card, D. and A.B. Krueger. 1996. "Labor Market Effects of School Quality: Theory and Evidence", Working Paper NBER Nr. 5450.

Cho, I.K. and D.M. Kreps. 1987. "Signaling Games and Stable Equilibria," Quarterly Journal of Economics, 102:2, pp. 179-221.

Cho I.K. and J. Sobel. 1990. "Strategic Stability and Uniqueness in Signaling Games," Journal of Economic Theory, 50:2. pp. 381-413.

Engers, M. 1987. "Signaling with Many Signals," Econometrica, 55:3, pp. 663-674.

Kreps, D.M. and J. Sobel. 1994. "Signalling," in Handbook of Game Theory. Aumann and Hart Eds. North Holland, 2, pp. 850-866.

Myerson, R.B. 1994. "Communication, Correlated Equilibria and Incentive Compatibility," in Handbook of Game Theory, Aumann and Hart Eds. North Holland, 2, pp.828-847. 
Noldeke, G. and E. Van Damme. 1990. "Signalling in a Dynamic Labour Market," Review of Economic Studies, 57:1, pp. 1-23.

Spence, A.M. 1973. "Job Market Signaling," Quarterly Journal of Economics, 87:3, pp. 355-374.

Spence, A.M. 1974. Market Signaling, Cambridge: Harvard University Press.

Stiglitz, J.E. 1975. "The Theory of "Screening", Education, and the Distribution of Income," American Economic Review, 65:3, pp. 283-300.

Van Damme, E. 1987. "Stability and Perfection of Nash Equilibria," Berlin: Springer Verlag.

Weiss, A. 1995. "Human capital vs. Signalling Explanations of Wages," Journal of Economic Perspective, 9:4, pp. 133-154. 